\title{
ZÜCHTUNG VON ORIENTIERTEN LITHIUMFLUORID-EINKRISTALLEN AUS DER DAMPFPHASE
}

\author{
W. O. MORITZ und D. WOLF \\ Institut für Kristallographie und Mineralogie der Universität München, D8 München 2, Germany
}

Received 2 March 1970

\begin{abstract}
A high vacuum oven was constructed to grow LiF single crystals from the vapor phase by the vertical pulling method. Using a special crucible top it is possible to grow oriented single crystals of $23 \mathrm{~mm}$ diameter and $70 \mathrm{~mm}$ length. At a temperature of about $150^{\circ} \mathrm{C}$ above the melting point the maximum pulling rate was
\end{abstract}

\section{Einleitung}

Beugungsuntersuchungen mit niederenergetischen Elektronen an Einkristalloberflächen von Alkalihalogeniden erfordern Kristalle hoher Reinheit.

Gute Spaltflächen sind eine notwendige Voraussetzung für einwandfreie Beugungsdiagramme, beşonders dann, wenn genaue Reflexintensitäten gemessen werden sollen.

Verunreinigungen an der Oberfläche - im Beugungsdiagramm als inkohärenter Untergrund bzw. als Überstrukturreflexe sichtbar - sind bei diesen Untersuchungen sehr störend.

Die Adsorption von Gasatomen wird durch die Anwendung von Ultrahochvakuum weitgehend vermieden; die Diffusion von Fremdatomen aus dem Kristallinnern kann durch Verwendung hochreiner Kristalle genügend herabgesetzt werden. Zur Herstellung geeigneter Kristalle bieten sich dabei mehrere Verfahren an.

Nach Recker ${ }^{1}$ ) können spannungsfreie Kristalle von Alkali - bzw. Erdalkalihalogeniden guter Reinheit und geringer Fehlstellenkonzentration aus der Dampfphase gezogen werden. Die Möglichkeit, eine Reinigung des Ausgangsmaterials und die Kristallzüchtung in der gleichen Apparatur durchführen zu können, ist ein Vorteil dieses Verfahrens. Die Behandlung des Ausgangsmaterials in gereinigter Fluorwasserstoff-Atmosphäre sowie ein Zonenreinigungsverfahren entfallen.

Zur Züchtung von LiF-Einkristallen wurde ein found to be $8 \mathrm{~mm} / \mathrm{h}$.

The purification effect of the sublimation was investigated for $\mathrm{Na}^{+}-, \mathrm{K}^{+}-, \mathrm{Ca}^{++}$-ions and it was found that the concentration of these impurities is of the order of $10^{-6}$, except for sodium for which the purification is much less effective.

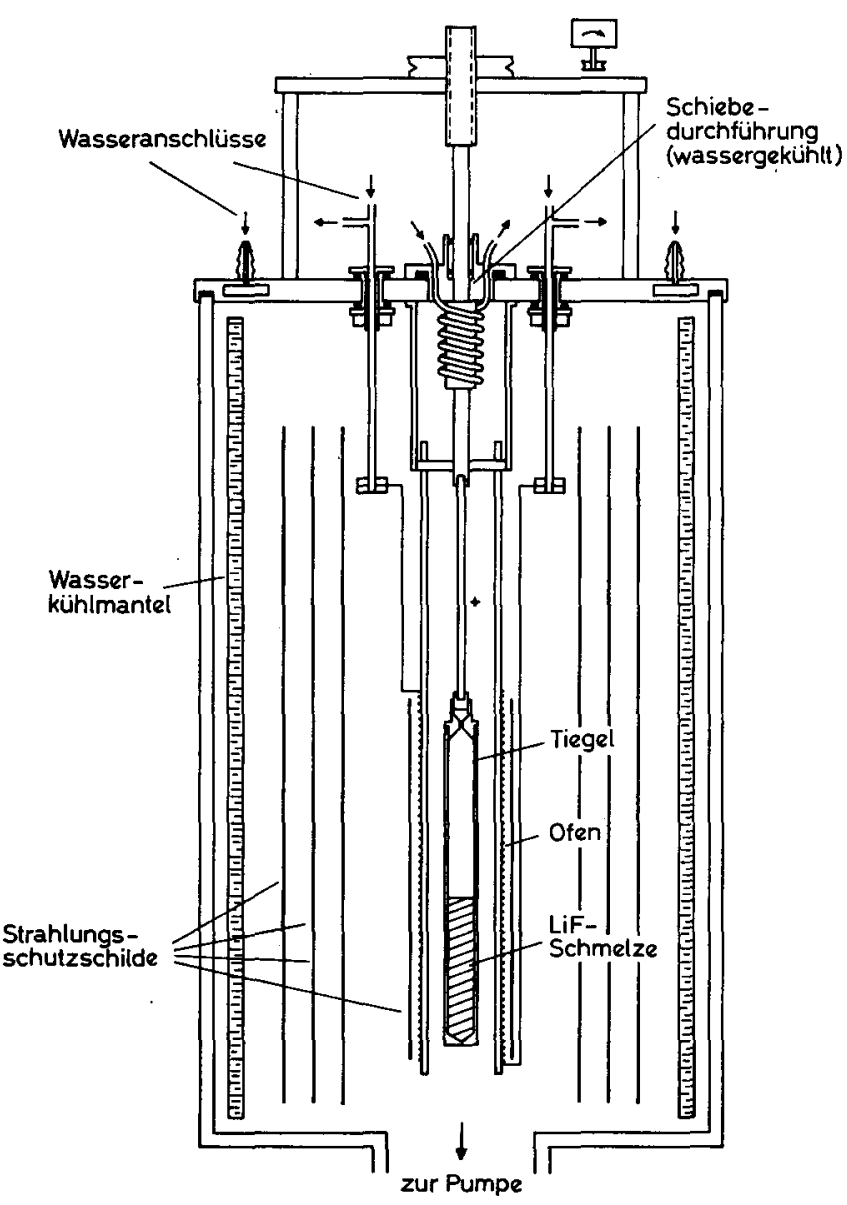

Abb. 1. Hochvakuumofen mit Zieheinrichtung und Tiegel in Ausgangsstellung. 
einfacher Ziehofen für den Einsatz in einem Hochvakuumrezipienten entwickelt.

\section{Beschreibung der Apparatur}

Eine schematische Darstellung der Züchtungseinrichtung zeigt Abb. 1. Heizofen und Ziehmechanik sind auf dem Deckflansch eines wassergekühlten Hochvakuumrezipienten montiert. Das auswechselbare Heizelement besteht aus einem AluminiumoxidKeramikrohr von $30 \mathrm{~mm}$ Innendurchmesser mit einer Heizwicklung aus Wolframdraht; Heizelemente eines anderen Temperaturprofils können leicht eingesetzt werden. Die Ziehachse läuft in einer vakuumdichten und ofenseitig wassergekühlten Simmerkammer; Vakuumundichtigkeiten durch zu starke Erwärmung der Ziehachse werden so zuverlässig verhindert.

Der dreiteilige Tiegel - Abb. 2 - besteht aus Reaktor-

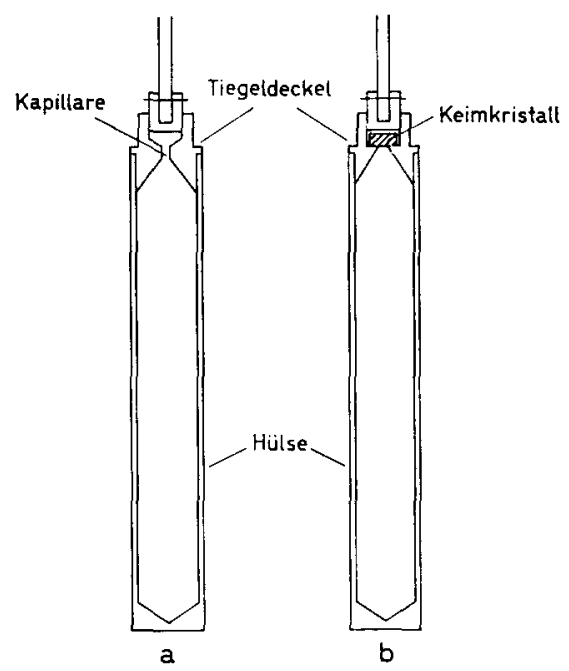

Abb. 2. Tiegel zur Züchtung von LiF-Einkristallen. (a) Keimauslese durch Kapillare, (b) orientiertes Kristallwachstum.

graphit. Bei Verwendung von dünnwandigen Tiegeln Wandstärken von $1.5 \mathrm{~mm}$ bei $25 \mathrm{~mm}$ Tiegeldurchmesser sind technisch möglich - wird der Temperaturgradient im Ofen nur unwesentlich verändert.

Die Form des Tiegeldeckels - Abb. 2a und 2b - ist entscheidend für Keimbildung und - auswahl bzw. Orientierung der Kristalle. Die Keimauswahl erfolgt beim Tiegeldeckel 1 - Abb. 2a - in einer Kapillare von 1.2-1.5 mm Durchmesser und $5 \mathrm{~mm}$ Länge. Die Kapillare endet in einer Erweiterung, die von einem Stopfen verschlossen wird. Un eine gute Entgasung des Innenraums zu erreichen, werden alle Teile nur lose zusammengesteckt und durch einfache Klammern aus Molybdändraht zusammengehalten.

Abb. 3 demonstriert Keimauswahl und beginnendes

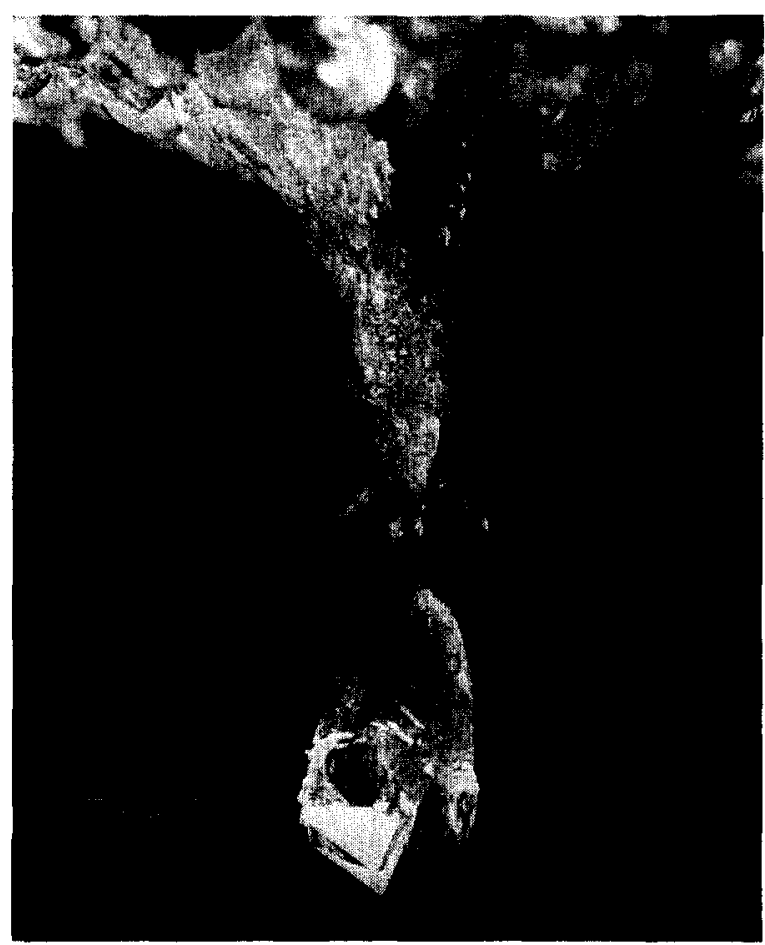

Abb. 3. Aussonderung eines Keimkristalls aus polykristallinem Niederschlag.

Kristallwachstum in der Kapillare. Zunächst bildet sich am Stopfen ein polykristalliner Niederschlag, aus dem sich beim Weiterwachsen durch die Kapillare anfänglich mehrere Keime, dann ein einzelner aussondern. Aus diesem Keim wächst ein Einkristall, dessen [111]-Richtung im allgemeinen einige Grad von der Ziehrichtung abweicht. Für die Verwendung in einem Spaltmanipulator wurden nach [100] orientierte Kristalle von mindestens $40 \mathrm{~mm}$ Länge benötigt.

Der Einsatz eines Keimkristalls im Raum über der Kapillare (Abb. 2a) führte zu keinem Erfolg.

Der geringfügig abgeänderte Tiegeldeckel 2 (Abb. $2 b)$ ist für die Züchtung von orientierten Einkristallen geeignet. Der Keimkristall liegt hierbei unmittelbar über einer Öffnung von $1.5 \mathrm{~mm}$ Diam. am Ende des Konus. Die Kantenlänge des Keims beträgt inı allgemeinen 7-8 $\mathrm{mm}$; kleinere Keime verdampfen vollständig. 
Mit dieser Anordnung wurden zuverlässig nach [100] orientierte Kristalle von $60 \mathrm{~mm}$ Länge und $23 \mathrm{~mm}$ Diam. gezüchtet.

\section{Kristallziichtung}

Ausgangsmaterial für die Kristallzüchtung ist LiFPulver der Qualität rein*; dieses Pulver wird durch mehrfache Vakuumsublimation einer Vorreinigung unterworfen. Mit diesem vorgereinigten Lithiumfluorid tritt beim Anheizen des Ofens - nach etwa halbstündigem Abpumpen des Rezipienten bis $\mathrm{zu}$ einem Druck von $\leq 1 \times 10^{-5}$ Torr - nur eine geringe Vakuumverschlechterung ein. Bei Ziehbeginn - nach einstündigem Anheizen des Ofens - ist der Druck wieder bei $1 \times 10^{-5}$ Torr und sinkt während des Ziehens rasch in den unteren $10^{-6}$ Torr-Bereich.

Abb. 4 zeigt den Temperaturverlauf des Ofens und

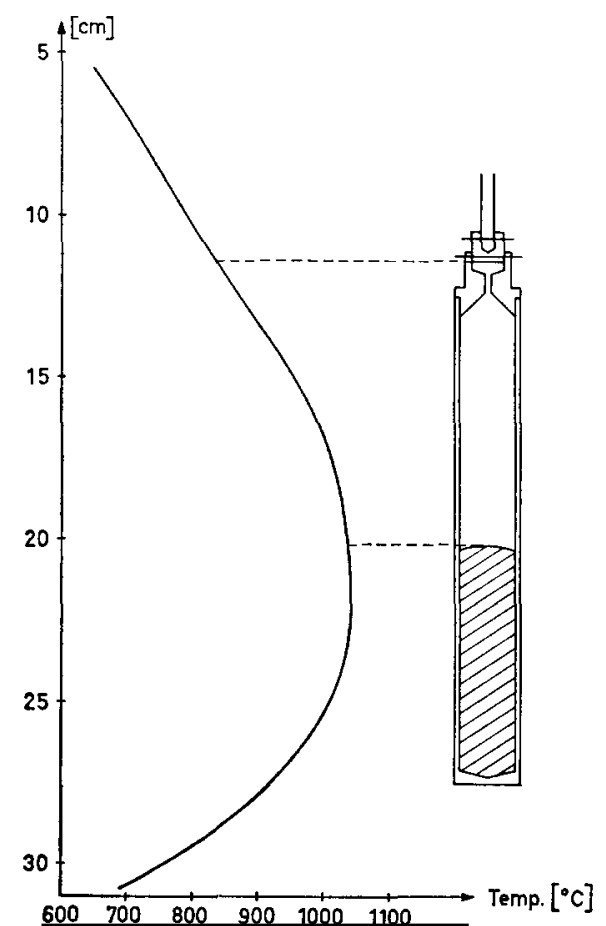

Abb. 4. Temperaturverlauf des Ofens mit Ausgangsstellung des Tiegels.

die Tiegelstellung bei Ziehbeginn. Die Temperatur und die Ziehgeschwindigkeit können in weiten Grenzen geändert werden. Bei einer Temperatur von $1000^{\circ} \mathrm{C}$ an der Oberfläche der Schmelze** beträgt die maximale

* Fa. Riedel de Haen.

** Schmelzpunkt von $\mathrm{LiF}=844^{\circ} \mathrm{C}$ nach Landolt-Börnstein.
Ziehgeschwindigkeit $8 \mathrm{~mm} / \mathrm{h}$, wobei der Temperaturgradient in der Wachstumszone $50^{\circ} \mathrm{C} / \mathrm{cm}$ ist. Für die
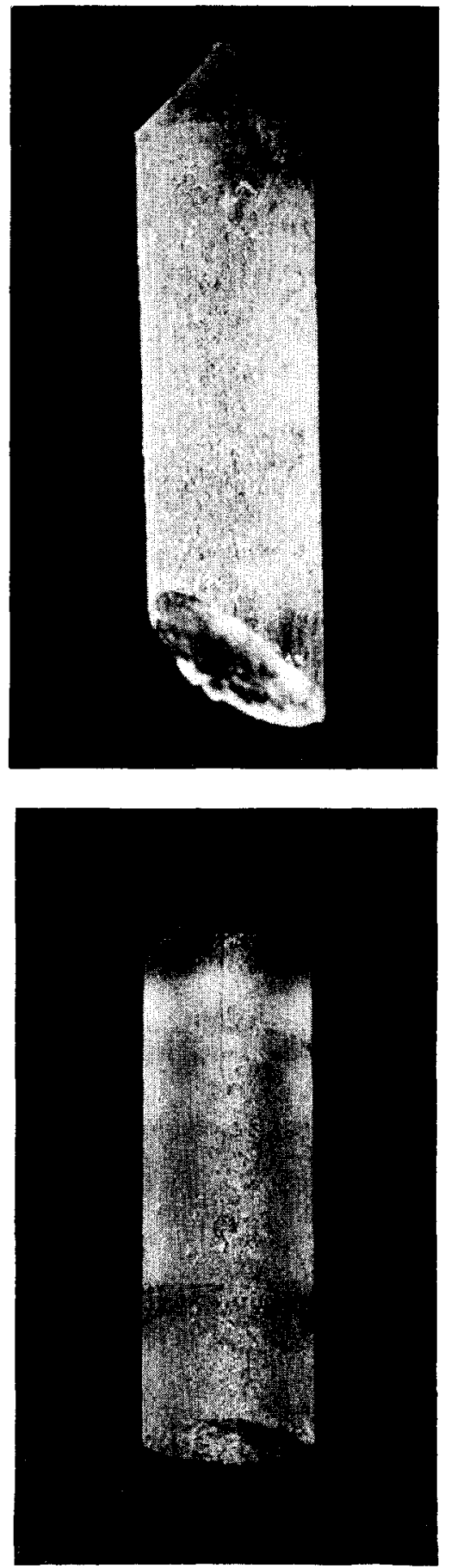

b

Abb. 5. Aus der Dampfphase gezüchtete Einkristalle (nat. Größe). (a) Zufällige Orientierung, (b) mit Keimkristall in Richtung [100] orientiert. 
Züchtung von orientierten Kristallen mit Keim ist bei einer Temperatur von $1000^{\circ} \mathrm{C}$ und einem Temperaturgradienten von $35^{\circ} \mathrm{C} / \mathrm{cm}$ eine Ziehgeschwindigkeit von $2.7 \mathrm{~mm} / \mathrm{h}$ günstig.

Die Ofentemperatur wird allein durch Einregeln des Heizstroms am Transformator eingestellt. Die damit erreichte Temperaturgenauigkeit und -konstanz während des Ziehvorgangs ist ausreichend; auf eine zusätzliche Temperaturregelung kann daher verzichtet werden.

Nach Beendigung des Ziehens wird die Temperatur mit einer Geschwindigkeit von $200{ }^{\circ} \mathrm{C} / \mathrm{h}$ abgesenkt; weiteres Tempern erübrigt sich für LiF-Kristalle.

\section{Ergebnisse}

Einen orientierten bzw. nicht orientierten Einkristall
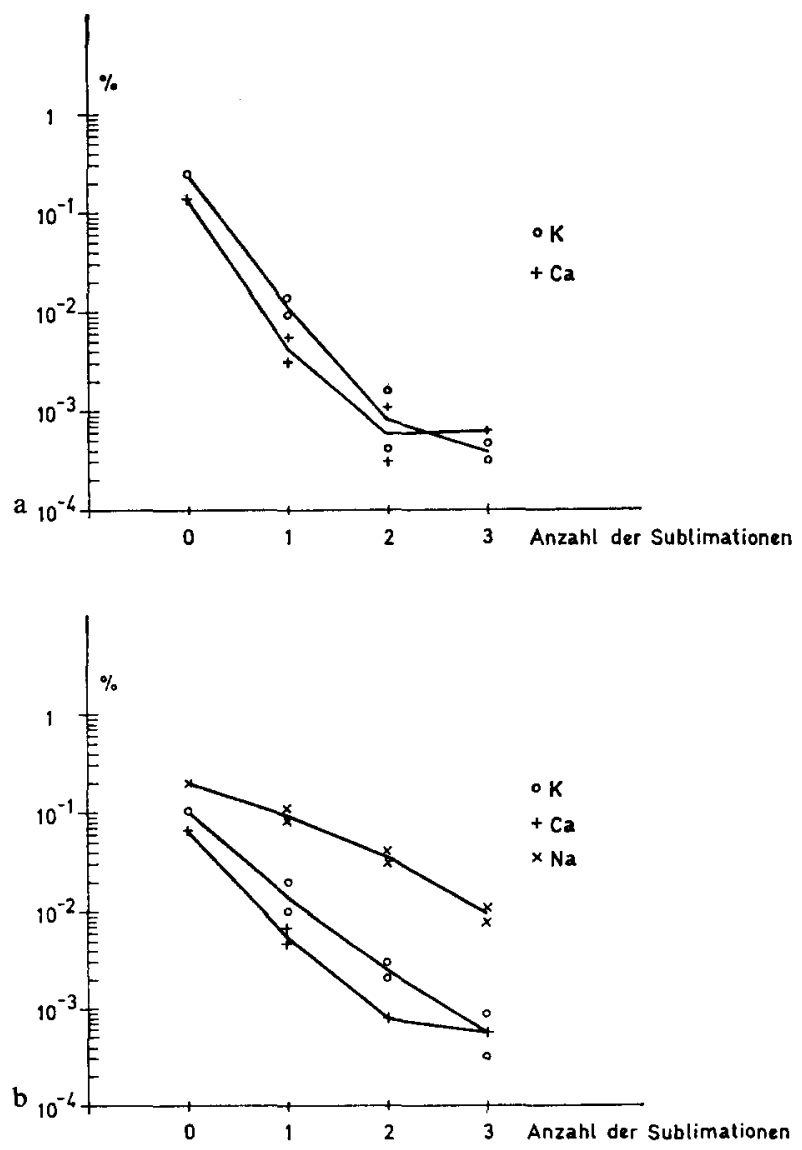

Abb. 6. Abnahme der Verunreinigung durch wiederholte Sublimation. (a) Untersuchung durch Röntgenfluoreszenzanalyse, (b) flammenspektrometrisch. Messung jeweils am Anfang und am Ende eines Kristalls. von $\mathrm{LiF}$ zeigt Abb. 5. Die Reinheit der Kristalle wurde durch Röntgenfluoreszenzanalyse bzw. durch flammenspektroskopische Untersuchungen bestimmt. Jeder Sublimationsschritt bedeutet einen Reinheitsgewinn um den Faktor 5-10 (Abb. 6a, 6b). Nach drei Sublimationsschritten ist ein Reinheitsgrad von $5 \times 10^{-4} \%$ für Kalium und Calzium und $10^{-2} \%$ für Natrium erreicht, gegenüber Werten des Ausgangsmaterials mit $0.1 \%$ für Kalium bzw. Calzium und $0.3 \%$ für Natrium. Die Verunreinigung am Kristallanfang ist im allgemeinen geringfügig grösser als am Kristallende.

Mit Ätzversuchen ${ }^{2}$ ) wurden die Versetzungsdichten bestimmt. Dabei wurde eine deutliche Abhängigkeit der Versetzungsdichten von den Ziehbedingungen festgestellt. Bei günstigen Ziehbedingungen - kleine Ziehgeschwindigkeit, niedrige Temperatur - wurden Versetzungsdichten von $10^{4} \mathrm{~cm}^{-2}$ erreicht.

\section{Zusammenfassung}

Für die Züchtung von LiF-Kristallen bzw. anderer Fluoride, z.B. $\mathrm{MgF}_{2}, \mathrm{CaF}_{2}, \mathrm{MnF}_{2}$, aus der Dampfphase wurde ein einfacher Hochvakuumofen aufgebaut. Durch eine spezielle Tiegelkonstruktion konnten orientierte Einkristalle von $23 \mathrm{~mm}$ Diam. und $70 \mathrm{~mm}$ Länge hergestellt werden. Die erreichte Ziehgeschwindigkeit beträgt bis zu $8 \mathrm{~mm} / \mathrm{h}$, stellt jedoch bislang kein Maximum dar. Durch mehrfache Vakuumsublimation wird das technische reine Ausgangsmaterial gereinigt. Die erreichten Reinheitsgrade für Alkali- bzw. Erdalkaliionen liegen in der Grössenordnung $10^{-6}$. Durch fortgesetzte Sublimationsreinigung sollte eine weitere Verbesserung der Reinheit möglich sein.

Weitere Untersuchungen werden durchgeführt.

Herrn Prof. Dr. H. Jagodzinski danken wir für die Anregung und Unterstützung dieser Arbeit. Herrn Dipl. Geol. Weber-Diefenbach danken wir für die Durchführung der Röntgenfluoreszenzanalyse, Frau G. Cammann für die flammenspektroskopischen Untersuchungen, Frl. A. Schmid für die Hilfe bei der Herstellung der Zeichnungen und des Manuskripts.

\section{Literatur}

1) K. Recker und R. Leckebusch, J. Crystal Growth 5 (1969) 125.

2) J. J. Gilman, W. G. Johnston und G. W. Sears, J. Appl. Phys. 29 (1958) 747. 